\title{
Die Wissenschaft als Wegbegleiterin zur Umsetzung der Leitlinien Deutscher Arktispolitik
}

\author{
Sebastian Leskien \\ Wirtschafts- und Sozialwissenschaftliche Fakultät, Universität Potsdam, Potsdam, Deutschland \\ Correspondence: Sebastian Leskien (sebastian.leskien@outlook.de)
}

Published: 20 May 2021

Kurzfassung. On 21 August 2019 the German Federal Cabinet stipulated its New Arctic Policy Guidelines. Science is an integral part of this political document according to which scientific findings shall be the starting point of the German activities in the Arctic. The present article argues that an independent "German" Arctic policy can only be successfully shaped by taking scientific knowledge into consideration. In order to investigate the role of science in German Arctic policy different governmental documents have been analyzed, notably the New German Arctic Policy Guidelines.

First of all, the article discusses why Germany as a NonArctic riparian state is pursuing its own Arctic policy. For this purpose, it considers the main reasons which have caused

Germany to exert an influence on the Arctic. These include strategic, economic, ecologic and especially researchoriented interests. Concerning the implementation of the goals of the New Arctic Policy Guidelines the knowledge transfer between science and politics is of special significance. The article describes how science contributes to political decisions by generating explanation models, different approaches to solutions or by issuing recommendations. In order to tackle the multi-layered and complex structured goals of the German Arctic Guidelines politics need to take into account research and science to remain capable of acting. Thus, science is indispensable for the realisation of the German Arctic Policy goals.

Finally, the article points out that the traditional polar research system is shifting from a traditional natural scientificbased orientation to a more inclusive and broad-ranging orientation. It shows how diverse academic disciplines scientifically deal with questions regarding the Arctic. Here, the article argues that the traditional natural scientific-based orientation of polar science is outdated whereas a demand for interdisciplinary Arctic polar science has emerged. By loo- king at the project "Marine Conservation in the Arctic" the article illustrates how these different academic fields can successfully be merged.

\section{Vorwort}

In den vergangenen Jahren hat sich die Arktis in der öffentlichen Wahrnehmung von einer „Randregion“ zu einer „Schlüsselregion der Weltpolitik“ (Deutscher Bundestag, 2019) gewandelt. Insbesondere das letzte Internationale Polarjahr 2007-2008 gab zahlreichen Nationen den Anstoß, Ziele für eine eigene Arktispolitik zu setzen (McCallum, 2018). Zu jenen Nationen zählte auch Deutschland, das 2013 erstmals und sodann 2019 in überarbeiteter Form, die ,neuen" Leitlinien deutscher Arktispolitik (Abb. 1) formulierte mit Entwicklungszielen und Handlungsansätzen für eine eigene deutsche Arktispolitik (Auswärtiges Amt, 2019). Obwohl es sich bei den neuen Leitlinien zunächst um eine überarbeitete Neuauflage der 2013 vom Auswärtigen Amt veröffentlichten Fassung handelt, waren an deren Novellierung erstmals die Bundesregierung sowie sieben weitere Bundesministerien ${ }^{1}$ gemeinsam beteiligt - eine ressortübergreifende Ausarbeitung. Ähnlich wie in dieser Erstausgabe werden die Freiheit der Meere, der Umweltschutz, wirtschaftliche Interessen sowie die Erforschung der Arktis betont. Die Wissenschaft nimmt in diesem politischen Dokument jedoch eine hervorgehobene Rolle ein, wonach ,wissenschaftliche Erkenntnisse der Ausgangspunkt für das Handeln Deutschlands im arktischen Raum“ (Auswärtiges Amt, 2019) sein sollen.

\footnotetext{
${ }^{1}$ Hierbei handelt es sich um folgende Ressorts: Ernährung und Landwirtschaft (BMEL), Forschung (BMBF), Umwelt (BMU), Verkehr (BMVI), Verteidigung (BMVg), Wirtschaft (BMWi), Wirtschaftliche Zusammenarbeit und Entwicklung (BMZ).
} 


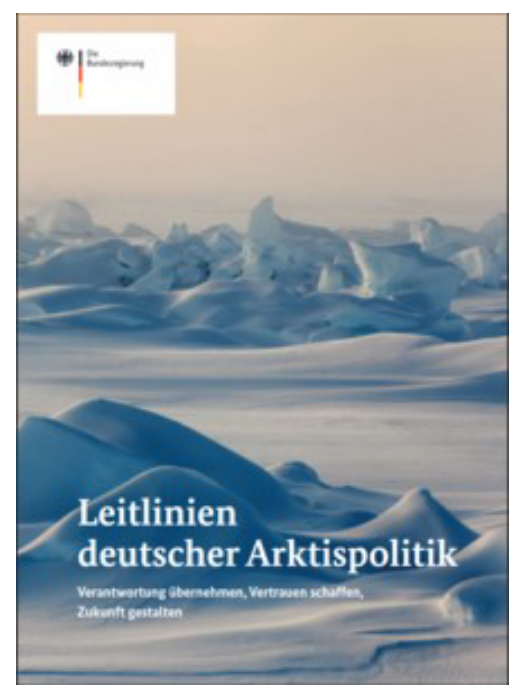

Abb. 1. Titelseite der „Leitlinien deutscher Arktispolitik“ der Bundesregierung, veröffentlicht am 21. August 2019 (Bildquelle: Meereisportal, 2019. Kurzmeldung vom 30. August 2019).

Vorliegender Beitrag soll zum Verständnis beitragen, welche Schlüsselrolle der Wissenschaft in der Umsetzung der Leitlinien deutscher Arktispolitik zukommt. Es wird argumentiert, dass nur durch das Einwirken von Wissenschaft eine eigene ,deutsche“ Arktispolitik erfolgreich gestaltet werden kann. Des Weiteren hinterfragt die Arbeit das Bestehende polarwissenschaftliche Forschungssystem und betont die Notwendigkeit eines fachübergreifenden Austausches.

Wissenschaft wird hierbei begriffen als die Vielfalt des nach Erkenntnisgewinn strebenden Fächerkanons, die gleichwohl in der Lage ist, ihre Forschungsergebnisse zur sinnvollen und nachvollziehbaren Erklärung bestimmter $\mathrm{Zu}-$ sammenhänge oder Phänomene bereitzustellen (LenhardSchramm, 2012) und dabei gleichzeitig einen Gestaltungsanspruch verfolgt (Rost, 1966).

\section{Warum hat Deutschland eine eigene Arktispolitik?}

Obwohl Deutschlands nördlichster Landpunkt Alemböög (Sylt) etwa $1224.5 \mathrm{~km}$ südlich des 66. Breitengrades, ${ }^{2} \mathrm{dem}$ Nördlichen Polarkreis, liegt und Deutschland somit im Verständnis des Seerechtsübereinkommens (SRÜ) der Vereinten Nationen nicht als arktischer Anrainerstaat gilt, findet die Arktis seit $1969^{3}$ dennoch Beachtung in der Außenpolitik der Bundesrepublik (Deutscher Bundestag, 1969). Die Mo-

\footnotetext{
${ }^{2}$ Die hier verwendete Koordinate für den nördlichen Polarkreis lautet $66^{\circ} 33^{\prime} 43$ N. Für Alemböög die Koordinate $55^{\circ} 3^{\prime} 8 \mathrm{~N}$. Berechnet mithilfe von https://rechneronline.de/geo-koordinaten/ \#entfernung (letzter Zugriff: 17. Februar 2021).

${ }^{3}$ Jahr 1969: Teilnahme der deutschen Delegation an der Nordatlantischen Versammlung, in welcher die Erforschung der arktischen Gewässer empfohlen wurde.
}

tivation, eigene Impulse zur Gestaltung des Hohen Nordens $\mathrm{zu}$ setzen, wird dabei von verschiedenen nationalen Begehrlichkeiten flankiert:

Forschungsinteressen. Deutschland kann auf eine über 150-jährige polare Forschungsgeschichte zurückblicken. Bereits im 19. Jh. waren es vor allem deutsche Wissenschaftler (u.a. Carl Koldewey, August Petermann), welche zu einer ersten kartografischen Erfassung von Teilen des Hohen Nordens beigetragen haben (Venzke, 1988). Gegenwärtig sind es vor allem Meilensteine wie die MOSAIC-Expedition oder die wissenschaftlichen Erkenntnisse des AWIs mit denen sich Deutschland weltweit erfolgreich in der Arktisforschung profiliert. Die in Forschungsvorhaben gesammelten Ergebnisse sind laut der neuen Arktisleitlinien insbesondere für politische Handlungen relevant, wonach ,alle arktispolitischen Entscheidungen erst auf der Basis von Erkenntnissen einer „,freien“ und ,verantwortungsvollen“ Forschung getroffen werden sollen (Auswärtiges Amt, 2019). Zudem definieren die Leitlinien Deutschland vor allem aufgrund seines starken Profils in der Polarforschung als ,internationalen Akteur im Hohen Norden“ (Auswärtiges Amt 2019). Die heutige Relevanz der Polarforschung für die Bundesrepublik spiegelt sich u.a. auch in der Forschungsförderung wider. So finanzierte Deutschland zwischen 2007-2016 mit insgesamt 250 arktisbezogenen Forschungsprojekten mehr Vorhaben als mancher der arktischen Anrainerstaaten (z.B. Finnland, Schweden) (Osipov et al., 2017).

Ökonomische Interessen. Eine sich im Nordpolarmeer rapide vollziehende Klimaerwärmung lässt Meereis schmelzen und neue Seewege entstehen, die Pazifik und Atlantik nördlich der Kontinente verbindet (Abb. 2: Deutsches Arktisbüro, 2019a). Als Industrieland mit einem Umsatz von EUR 511,1 Mrd. (2018) durch den Seehandel, hat Deutschland allerdings ein Interesse daran, durch neue maritime Infrastrukturen (Nordwest- und Nordostpassage) in der Arktis, den Wirtschaftsverkehr mit seinem wichtigsten Handelspartner, China, zu intensivieren (Hansa, 2019). Schätzungen gehen davon aus, dass eine Schifffahrt zwischen dem Hamburger Hafen und Shanghai entlang der rund $6500 \mathrm{~km}$ langen Nordküste Russlands eine Zeitersparnis von einer Woche gegenüber der traditionellen Schiffsroute über den Suezkanal bedeutet (Görner, 2018).

Ökologische Interessen. Die globale Erwärmung vollzieht sich durch den Albedo-Effekt in der Arktis gleich doppelt schneller als im Vergleich zum globalen Mittelwert (IPCC, 2019). Die Auswirkungen dieser Veränderungen (u.a. Auftauen der Permafrostböden, Anstieg des Meeresspiegels durch Schmelzwasser, Versauerung der Ozeane) sind jedoch nicht nur regional in der Arktis wahrnehmbar, sondern werden nach Einschätzung von Klimaforschern auch in anderen Weltregionen, darunter Deutschland, langfristig spürbar werden (EEA, 2019). Gerade weil die Arktis über Atmosphäre und Meeresströmungen mit den übrigen Erdteilen verbunden ist, hat Deutschland ein großes Eigeninteres- 


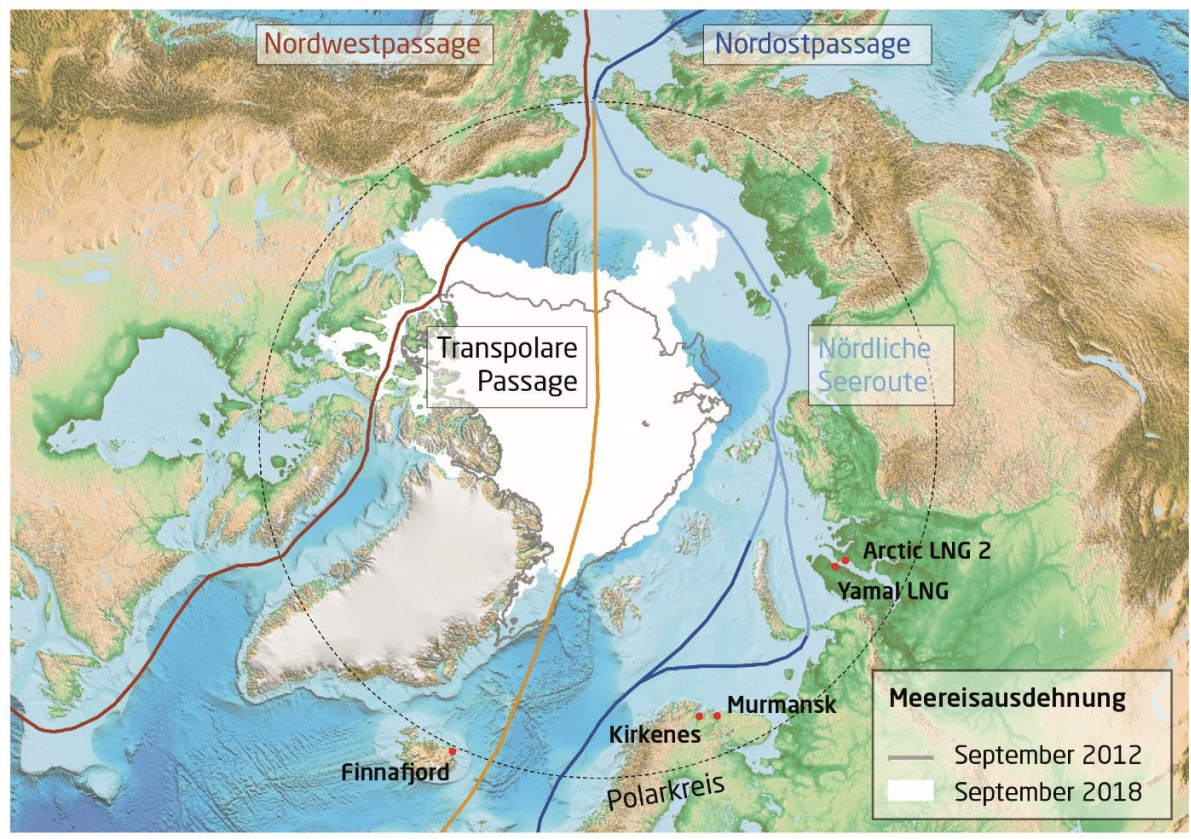

Abb. 2. Drei Routen kommen für eine Durchquerung des Nordpolarmeeres in Frage. Die Nordostpassage entlang der russischen und norwegischen Küste ist aufgrund der günstigeren Eisbedingungen und besseren Beschiffbarkeit im Vergleich zur Nordwestpassage und Transpolaren Passage zurzeit die aussichtsreichste Route (Quelle: Deutsches Arktisbüro (2019a): Fact Sheet. Schifffahrt in der Arktis).

se am Schutz und Erhalt der natürlichen Umwelt der Arktis (Maribus, 2019; Notz, 2011).

Kooperationen und Sicherheit. Die sicherheitspolitische Diskussion um den Hohen Norden ist durch Konfliktfelder wie territoriale Fragen, Ressourcengewinnung und Seewegnutzung zwischen den Arctic Five (Kanada, Königreich Dänemark, Norwegen, die Russische Föderation, Vereinigte Staaten von Amerika) ${ }^{4}$ bestimmt. Gespräche zwischen diesen Staaten zur Nutzung und Entwicklung sind daher vom Einsatz vertrauensbildender Maßnahmen begleitet, um die arktische Region als gewaltfreie, friedliche Zone multilateraler Zusammenarbeit zu erhalten. Wichtigste Austauschplattform ist der Arktische Rat, einem zwischenstaatlichen Forum, welches sich ausschließlich mit arktischen Themen befasst (Deutsches Arktisbüro, 2019b). Deutschland ist seit 1996 als ständiger Beobachterstaat Mitglied im Arktischen Rat und hat ein hohes Eigeninteresse an dem Erhalt von Stabilität und Kooperation im Nordpolarmeer. Zum einen lassen sich erst unter friedlichen Bedingungen die wissenschaftlichen, ökologischen und ökonomischen Interessen Deutschlands in der Arktis bestmöglich verfolgen. Zum anderen strebt Deutschland für sich eine Vermittlerrolle im arktischen Akteursumfeld an (Auswärtiges Amt, 2019). In dieser kann es seine Position im Rat dazu nutzen, um seine eigene Rolle in den internationalen Arktisbeziehungen zu sichern.

\footnotetext{
${ }^{4}$ Aufzählung gemäß ihrer Reihenfolge und Benennung im Arktischen Rat.
}

\section{Worum geht es in den Leitlinien?}

Die neuen Leitlinien deutscher Arktispolitik verfolgen einen ausgesprochen holistischen Aktionsansatz, d.h. es werden die Interdependenzen und Synergien von sicherheitspolitischen Fragen, Anliegen des Naturschutzes, Wirtschaftsförderung, Forschungsaktivitäten, internationale Kooperationen als auch die Verantwortung aller in der Region agierenden Akteure beleuchtet. Obwohl die neuen Leitlinien insbesondere den Umweltschutz (im Vergleich zur Ursprungsfassung von 2013) noch stärker in den Vordergrund rücken, sind sie keinesfalls ein Bekenntnis, die Arktis im Rahmen des deutschen Engagements , unangetastet“ zu lassen, sondern mithin auf einen ,nachhaltigen“, aber insbesondere „schonenden“" Umgang mit der Region hinzuwirken (Auswärtiges Amt, 2019). Hinsichtlich der Nutzung der Arktis ergeben sich aus den aktuellen Leitlinien folgende Ziele deutscher Arktispolitik:

- Erhalt arktischer Biodiversität durch Ausweitung von Schutzzonen und verstärkte nationale Beteiligung in entsprechenden Naturschutzprojekten,

- Reduzierung der arktischen Umweltverschmutzung durch den Einsatz neuer Technologien, Treibstoffe bei gleichzeitiger Förderung von verantwortungsvollem Handeln in allen Nutzungsbereichen (Tourismus, Fischerei, Güterschifffahrt, Forschung), 
- Nutzung des ökonomischen Potentials der Arktis (u.a. durch die Exploration und den Abbau von Bodenressourcen),

- Stärkung multilateraler Kooperationen zur Lösung sich überschneidender Nutzungsinteressen,

- Schutz und Anerkennung der Rechte und Freiheiten von Indigenen durch Wahrung ihres Lebensraums sowie

- Förderung der Arktisforschung durch Sicherstellung der Zugänglichkeit der arktischen Gebiete für Forschungsvorhaben.

Darüber hinaus werden die deutschen Nutzungsinteressen in der Arktis determiniert durch die Hervorhebung des Vorsorge- und Verursacherprinzips als Grundlage für alle wirtschaftlichen und umweltpolitischen Aktivitäten sowie der Einhaltung internationaler Rahmenregelungen, wie etwa des Pariser Klimaabkommens, des Internationalen Seerechtsübereinkommens (SRÜ) oder des Polar Codes.

\section{Welche strategische Schlüsselrolle nimmt die Wissenschaft bei der Verwirklichung der Arktisleitlinien ein?}

Wissenschaftliche Erkenntnisse haben für politische Entscheidungen einen grundlegenden Stellenwert. Um gesellschaftliche Kontexte zu regeln, Konflikte auszugleichen aber auch um (inner-)politische Auseinandersetzungen für sich zu entscheiden, nehmen Politiker*innen zur Sicherung der Legitimation ihrer Entscheidungen regelmäßig Rückgriff auf den Sachverstand von Expert*innen (Voßkuhle, 2008). Gleichzeitig profitieren auch Forschende in erheblichem MaBe von der Wahrnehmung ihrer Arbeit in Form von Anerkennung durch Politikvertretende, Forschungsförderung und im Idealfall einem gesellschaftlichen „Impact“ ihrer Leistungen. Auf der internationalen Ebene wird der operationelle Charakter dieser symbiotischen Beziehung in der Wissenschaftsdiplomatie deutlich. Diese bezeichnet den Einsatz und die Anwendung wissenschaftlicher Zusammenarbeit zur Förderung des Brückenbaus und der Verbesserung der diplomatischen Beziehungen zwischen Staaten ${ }^{5}$ (Cordis, 2009). Sie ist somit als „soft power“-Komponente mittlerweile fester Bestandteil der Außenpolitik vieler Staaten und findet Ausdruck in zahlreichen wissenschaftlichen Kooperationsprojekten in denen über Dialoge zwischen Nationen und Kulturen gemeinsame Ziele und Anliegen thematisiert werden. Aus deutscher Sicht ist der Dialog zwischen Wissenschaftler*innen (und mit Politiker*innen) in dem wachsenden Akteurs- und Wirkungsumfeld der Arktis unerlässlich, um auf die Zielerfüllung der Leitlinien deutscher Arktispolitik hinzuwirken (Grosfeld et al., 2019). Es haben sich z.B. Formate wie der

\footnotetext{
${ }^{5}$ Definition angelehnt an Vaughan Turekian, Direktor des Zentrums für Wissenschaftsdiplomatie, in: Cordis (2009)
}

Arktisdialog oder (auf internationaler Ebene) die Arktische Wissenschaftsministerkonferenz, die Deutschland 2018 ausgerichtet hat, etabliert, in denen vor allem ein Wissenstransfer im Vordergrund steht. In diesem Transfer kommt der Wissenschaft als Urheberin von neuem Wissen eine Schlüsselrolle zu. Durch die Generierung und die Bereitstellung von Systemwissen (Wissen über das Zustandekommen und die potentielle Entwicklung eines bestimmten Ist-Zustandes), Ziel- bzw. Orientierungswissen (Wissen darüber, welche Ziele mit der Veränderung eines bestimmten Ist-Zustandes angestrebt werden sollen) und Transformationswissen (Wissen über den Weg vom Ist- zum Ziel-Zustand) (Pohl und Hirsch Hadorn, 2006) liefert Wissenschaft steuerungsrelevante Erklärungs- bzw. Lösungsansätze sowie Empfehlungen zu bislang wenig erforschten Fragen für verschiedene Anwender*innen (z.B. Politiker*innen, Behördenmitarbeitende, Medienvertretende). Gleichzeitig finden Rückkopplungen statt, denn wissenschaftliche Erkenntnisse werden nicht allein zu Praxiswissen, indem Forschung diese linear in die Praxis ,transferiert“. Vielmehr handelt es sich stattdessen um einen höchst diskursiven und rekursiven Prozess, bei dem die Generierung, die Modifikation, die Bewertung sowie die Weitergabe von Wissen ineinandergreifen und schließlich zu einer Rekombination von Wissen führen kann (Blotevogel und Wiegand, 2015). Diese Rekombination wird vor allem dann deutlich, wenn Forschende und praxisorientierte Akteur*innen (z.B. Politiker*innen, Behördenmitarbeitende) im Rahmen von Ko-Produktionsprozessen miteinander interagieren und anwendungsrelevantes Wissen erzeugen. Für einen erfolgreichen Wissenstransfer mit einem Bezug zur Arktis ist die Verknüpfung verschiedener wissenschaftlicher Perspektiven sowie die Einbeziehung ausgewählter Wissenschaftsakteure, die sich mit spezifischem Wissen zur Arktis auszeichnen, gleichfalls wichtig. So können verschiedene Wissenschaftsdisziplinen aufgrund ihrer vielfältigen Methoden und Fragestellungen unterschiedliches Wissen beitragen, um den Wissenstransfer zwischen Politik und Wissenschaft zu bereichern und darüber hinaus auf die vielfältigen Ziele der Arktisleitlinien, die allein aufgrund ihres Umfanges in der Praxis nicht nur durch eine Disziplin allein erfüllt werden können, hinwirken. Ein Beispiel bietet das in den deutschen Leitlinien häufig genannte Ziel der Verbesserung des arktischen Umweltschutzes. Dieses Ziel kann, je nach Disziplin, auf verschiedene Art und Weise bearbeitet werden. Während naturwissenschaftlich-analytische Erkenntnisse genutzt werden, um die komplexen physikalischen und biogeochemischen Wechselwirkungen in den arktischen Regionen erklärbar zu machen, leisten ingenieurswissenschaftlichtechnische Ausrichtungen dagegen ihren Beitrag zum arktischen Umweltschutz durch die Konstruktion entsprechender umwelttechnischer Anlagen (z.B. zur Reduzierung von Stoffeinträgen in arktische Gewässer). Einen ganz anderen Ansatz nutzen geistes- und sozialwissenschaftliche Fächer, die ethische oder finanzieller Entscheidungshilfen für die politische Praxis auf der Basis empirischer Untersuchungen 
formulieren (z.B. zur Nutzbarmachung traditionellen indigenen Wissens über die arktische Umwelt). Rechtswissenschaftliche Expertise ist hingegen in solchen Bereichen für den arktischen Umweltschutz wichtig, wenn Maßnahmen des Umweltschutzes (a) eine extraterritoriale Wirkung entfalten sollen, d.h. wenn zwischenstaatliche Abkommen notwendig werden, um eine gemeinsame Region im gleichen Territorium zu schützen oder (b) innerstaatliches Umwelthandeln eine Koordination oder Übertragung staatlicher Zuständigkeiten zwischen verschiedenen Organisationen bedarf (Bothe, 1972). Voraussetzung für eine solche fachliche Verknüpfung ist jedoch, dass Arktisforschung nicht mehr nur als eine Teildisziplin der naturwissenschaftlichen Polarforschung wahrgenommen werden darf. Stattdessen wird die Arktisforschung spätestens mit der Etablierung von GovernanceStrukturen im Hohen Norden, wie dem Arktischen Rat oder der zunehmenden Verrechtlichung von Nutzungsansprüchen im Nordpolarmeer, von verschiedenen Wissenschaftsdisziplinen betrachtet und ist mithin eine Integrationswissenschaft (hierzu auch Abb. 3: Arktisforschung als Integrationswissenschaft). Obgleich sie sich aus unterschiedlichen Fachdisziplinen mit eigenen Fragestellungen, Methoden und Hintergrundüberzeugungen subsumiert, behandeln alle Teilgebiete der Arktisforschung vor allem denselben Forschungsgegenstand im Kern: die Arktis. Dieses einende Element der verschiedenen Disziplinen könnte Ansatzpunkt sein, um die Disziplinen zukünftig stärker in einer gemeinsamen Forschung zu integrieren. Wie wichtig fachübergreifende Forschungsansätze sind, wurde bereits von Mittelstraß hervorgehoben, der von der „Einheit der Wissenschaft“" spricht, d.h. die Vorstellung, dass das wissenschaftliche System über eine bloße Ansammlung von Wissensbeständen oder die Aufzählung einzelner Disziplinen hinausgeht (Mittelstraß, 1989). Nach ihm sei ein fachübergreifendes Denken fundamental, weil ,,(1) die Welt als Gegenstand der Wissenschaft, eine ist (Einheit der Natur), (2) das wissenschaftliche Interesse eines ist (es geht um ein rationales Begreifen der Welt)“ (Mittelstraß, 1989, 60). Diese Gedanken knüpfen wiederum an die neuen Leitlinien deutscher Arktispolitik an. Obwohl die neuen Leitlinien deutscher Arktispolitik den heterogenen Charakter der Arktis mit seinen vielfältigen Lebewesen, indigenen Völkern, natürlichen Gegebenheiten, Ressourcen oder auch deren Rollen im Klimawandel hervorhebt, wird eine einzelne Disziplin kaum dazu in der Lage sein, das komplexe Wechselwirkungsumfeld der Arktis vollständig zu erfassen und alle Ziele gleichzeitig bearbeiten zu können. Vielmehr ist ein hoher Grad an Offenheit gegenüber unterschiedlichen Denkstilen und Wissensarten bei der Bewältigung arktisbezogener Fragen überhaupt erst notwendig (Pohl und Hirsch Hadorn, 2008), um diese detailliert bearbeiten zu können. Eine Offenheit gegenüber anderen Wissenschaften führt in der Praxis nicht nur zu mehr Verständnis füreinander, sondern erlaubt auch die besondere Chance punktuell einzelne Fragestellungen mit fachfremden Methoden detaillierter zu beantworten, wozu Einzelwissenschaften nicht möglich wären.
Speziell für die Arktisforschung wäre es gleichwohl denkbar, langfristig einen fachübergreifenden Arbeitskanon $\mathrm{zu}$ entwerfen, der eigene Zugangsweisen, Methoden, Regeln, Verbote oder empirische Settings beinhaltet, um das fachübergreifende Arbeiten positiv auszuweisen und $\mathrm{zu}$ typisieren (Schmidt, 2005). Eine gewisse Standardisierung wäre nicht zuletzt auch der Umsetzung der Ziele aus den neuen Arktisleitlinien ausgesprochen zuträglich, weil sich durch fachübergreifendes Arbeiten auch die Problemwahrnehmung erhöht und im Idealfall umfangreichere Lösungsmöglichkeiten formulieren lassen.

Aktuell durchgeführte Forschungsprojekte mit einem solchen fachübergreifenden Charakter zeigen bereits auf, dass moderne wissenschaftliche Arktisforschung trotz prinzipieller Leistungsgrenzen (z.B. unterschiedlicher methodischer Ansätze), durchaus erfolgreich sein können: Hierzu zählt etwa das vom Bundesamt für Naturschutz vergebene und vom IASS Potsdam, dem WWF sowie dem Ecologic-Institut umgesetzte Vorhaben „Meeresnaturschutz in der Arktis“. Die Partner erarbeiten gemeinsame naturschutzfachliche Vorschläge für die ,naturschutzrelevanten Arbeitsgruppen des Arktischen Rates“ mit dem langfristigen Ziel ein ,panarktisches Netzwerk mariner Schutzgebiete“ zu etablieren (Bundesamt für Naturschutz, 2018). Die fachübergreifende Arbeit äußert sich einerseits durch die an der Projektumsetzung beteiligten Akteure aus unterschiedlichen Wissensgebieten (Sozial-, Rechts- und Naturwissenschaften) als auch durch den Projektauftrag selbst. So soll die naturschutzfachliche Arbeit auf der Basis von ,internationalem Meeresnaturschutz, Ocean Governance und [... J Jurisdiktion, als auch über einschlägige Erfahrungen über die Arbeit des Arktischen Rates“ (Bundesamt für Naturschutz, 2018, 2) gestaltet werden.

Bei dem genannten Projekt handelt es sich jedoch um einen Präzedenzfall, der allerdings für die weitere Umsetzung der deutschen arktispolitischen Ziele durchaus Orientierung geben kann.

\section{Wer sind die Wissensträger*innen?}

Ein Blick auf die polare Forschungsarchitektur hierzulande erscheint hilfreich, um die unterschiedlichen Akteure und Organisationen der deutschen Arktisforschung zu identifizieren, die an der Umsetzung der Arktisleitlinien wesentlich beteiligt sind, z.B. im Rahmen von Politikberatung, der Bereitstellung von Daten aus eigenen wissenschaftlichen Feldforschungen oder der Einbringung technologischer Innovationen. Es ist dabei festzustellen, dass die deutsche Arktisforschung durch ein breites Netz verschiedener Institute aus nahezu allen Wissenschaftsdisziplinen wahrgenommen wird. Hierzu zählen allen voran Fachbehörden, Universitäten sowie außeruniversitäre Forschungseinrichtungen. Jeder der im Folgenden genannten Akteure verfügt zwar über spezifische Ressourcen und verfolgt eigene wissenschaftliche Schwer- 
Arktisforschung als Integrationswissenschaft

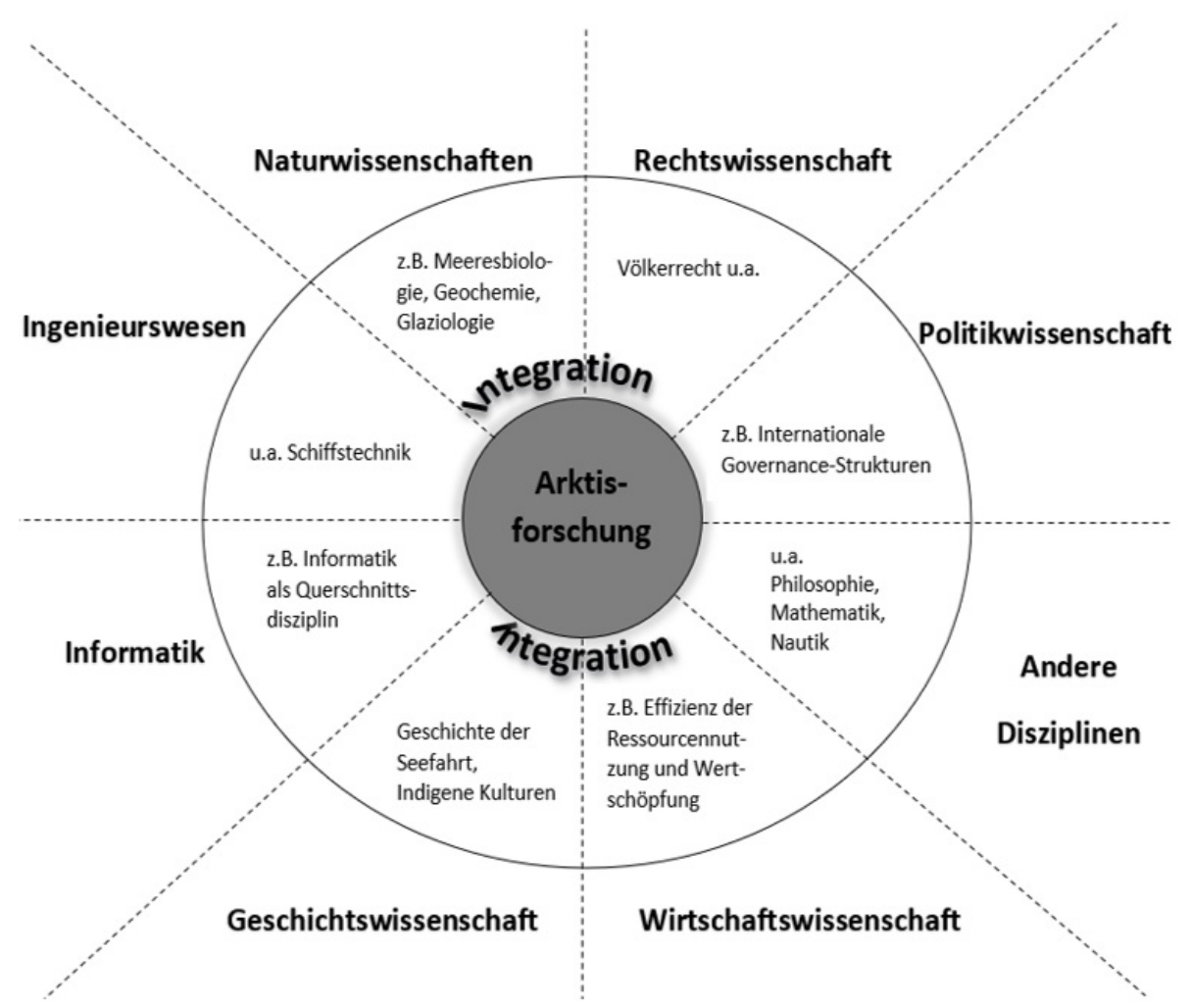

Abb. 3. Arktisforschung als Integrationswissenschaft (Eigene Darstellung).

punkte. Ihre große Gemeinsamkeit besteht jedoch in erster Linie im gemeinsamen Forschungsgegenstand, die Arktis wissenschaftliche zu erforschen und deren Zukunft im Rahmen der deutschen Arktisleitlinien mit zu gestalten:

Einen breiten Forschungsansatz verfolgt das AlfredWegener-Institut (AWI) als Helmholtz-Zentrum für Polarund Meeresforschung, welches seit 30 Jahren mithilfe von Forschungsstationen, Schiffen und Flugzeugen Untersuchungen zum Zusammenwirken von Mensch, Klima und Ozean in den Polarregionen durchführt. Ebenfalls veranstaltet das AWI seit 2013 den alle halbe Jahre stattfindenden Arktisdialog zum Informationsaustausch zwischen Polarforschung, Politik, Wirtschaft und Zivilgesellschaft zu aktuellen Arktisfragen. Die Veranstaltung wird hierbei inzwischen jeweils abwechselnd von einem der sieben Bundesministerien gastgebend mitbegleitet, die an der Konzeption der neuen Arktisleitlinien beteiligt waren und auf diese Weise thematisches Wissen aus ihren eigenen Ressorts einbringen. Die Koordination des Arktisdialoges übernimmt seit seiner Gründung 2017 das am AWI ansässige Deutsche Arktisbüro, welches 2018 auch die fachliche Vorbereitung der zweiten Arktischen Wissenschaftsministerkonferenz zur besseren internationalen Forschungszusammenarbeit übernommen hat. Des Weiteren ist das Deutsche Arktisbüro in die Begleitung von
Positionen zu Arktisfragen der Bundesregierung involviert und trägt durch seine beratende Tätigkeit maßgeblich zur Sichtbarkeit Deutschlands in der internationalen Arktispolitik bei.

Ebenfalls in Potsdam ansässig ist das Institut für transformative Nachhaltigkeitsforschung (IASS), welches seit 2009 im Rahmen verschiedener Projekte zu Fragen an der Schnittstelle von Nachhaltigkeit, Governance und Transformation der Arktis arbeitet. Die Forschungsbeiträge des IASS fließen insbesondere in die Arbeitsgruppe für nachhaltige Entwicklung des Arktischen Rates mit ein (IASS, 2020).

Einen ähnlichen Ansatz wie das IASS verfolgt auch das Ecologic Institut in Berlin, das naturschutzfachliche Beiträge zu aktuellen Problemlagen in der Arktis erarbeitet. So konzipierte das Institut in Kooperation mit dem Umweltbundesamt das Themenpapier „Umweltleitlinien deutscher Arktispolitik", deren Inhalte auch in den neuen Leitlinien deutscher Arktispolitik berücksichtigt wurden. Vom Ecologic Institut initiiert wurde das zwischen 2012-2018 stattfindende Arctic Summer College, eine politikorientierte Plattform zwischen Fachexpert*innen zum Austausch zu arktisspezifischen Themen (Ecologic Institute, 2020). Neben den außeruniversitären Forschungseinrichtungen gelangen Arktisthemen zunehmend auch auf den Lehrplan deutscher Hochschulen. An 
den Universitäten Bielefeld, Kiel und der Freien Universität Berlin lehren sozial- und geisteswissenschaftliche Polarforscher Studierende aus dem Bereich Politikwissenschaft, Philosophie und Geschichte. Die Arktis aus naturwissenschaftlicher Sicht hat dagegen bereits Platz auf dem Lehrplan verschiedener Universitäten, wie etwa denen in Rostock, Köln, Kiel, Bremen, Dresden, Trier und Leipzig, gefunden. Unter den Wissenschaftler*innen dieser Hochschulen engagieren sich (neben weiteren außeruniversitären Forschern) einige im Nationalkomitee für Polarforschung der Deutschen Forschungsgemeinschaft (DFG), welches die Aktivitäten der deutschen Hochschulen zur Arktisforschung gemeinsam mit dem AWI, dem Geomar Helmholtz-Zentrum für Ozeanforschung (GEOMAR) in Kiel sowie weiteren Bundeseinrichtungen und Instituten organisiert. Beachtenswert ist die im September 2017 erschienene „Polarforschungsagenda 2030“ zum heutigen Wissensstand der (deutschen) Polarforschung und den drängenden Forschungsfragen der Zukunft, welche unter der Leitung des Nationalkomitees erarbeitet wurde (siehe Heinemann et al., 2017).

Eine Kooperation in der Lehre erfolgt seit 2002 in Form des POMOR-Studiengangs (Polar and Marine Sciences), einem deutsch-russischen Kooperationsprojekt für Studierende an der Universität Sankt Petersburg und seinen beteiligten Partneruniversitäten in Bremen, Hamburg, Kiel, Potsdam sowie einigen Forschungseinrichtungen (AARI, AWI, GEOMAR, IOW). In dem Studiengang wird den teilnehmenden Studierenden fachübergreifendes Wissen zum arktischen Erd- und Klimasystem vermittelt. Aus deutscher Sicht stellt der POMOR-Studiengang im Bereich der Polarforschung ein ,,international sichtbares Aushängeschild der wissenschaftlichen Nachwuchsförderung dar" (Heinemann et al., 2017, 142).

Nicht zu unterschätzen ist der wissenschaftliche Impuls von Fachbehörden zur Umsetzung der neuen Leitlinien deutscher Arktispolitik. So sind die im Geschäftsbereich des Bundesumweltministeriums gehörenden Umweltbundesamt oder das Bundesamt für Naturschutz nicht nur an der Vergabe von Forschungsaufträgen beteiligt, sondern entwickeln auf der Basis von erhobenen Daten umweltrelevante Regelungen zum Schutz der Arktis, die sie in den Arbeitsgruppen des Arktischen Rates vorstellen (UBA, 2019). Die Bundesanstalt für Geowissenschaften und Rohstoffe (nachgeordnete Behörde des Bundeswirtschaftsministeriums) hat umfangreiche Informationen zum mineralischen Rohstoffpotential der Arktis zusammengetragen und gab hierzu eine Zusammenfassung heraus, deren Inhalte wegweisend für das ökonomische Interesse Deutschlands an der Arktis ist (Elsner et al., 2014).

Die Zusammenstellung aller Wissensträger*innen ist selbstverständlich nicht abschließend. In den letzten Jahren ist das nationale Interesse an der Arktis stetig gewachsen, sodass sich der Kreis der Akteure hierbei nur auf einige prominente Beispiele bezieht.

\section{Wie kann die Wissenschaft besser zur Erfüllung der Leitlinien beitragen?}

Obwohl die neuen Leitlinien deutscher Arktispolitik einen breiten Themenkanon aufgeschlagen haben, in dem alle Fachdisziplinen der Polarforschung anknüpfen können, dominieren im wissenschaftlichen Praxisalltag noch immer Einzeldisziplinen. Insbesondere der Brückenschlag zwischen naturwissenschaftlicher und sozialwissenschaftlicher Polarforschung ist erweiterungsfähig. Eine Mobilisierung der Wissenschaft ist jedoch gerade im Hinblick auf die Bewältigung der Ziele in den Leitlinien essentiell, da hinter jedem Ziel komplexe Fragestellungen, Handlungsfelder aber auch Zielkonflikte verborgen sind. Aus diesem Grund ist zu empfehlen, das Wissenschaftssystem insgesamt nutzbarer zu machen, indem zukünftig ein inter- oder transdisziplinärer Ansatz angestrebt wird.

Eine weitere Herausforderung ergibt sich vielmehr aus den politischen Rahmenbedingungen selbst. Bislang streben vier $^{6}$ der nordischen Staaten eine Erweiterung ihrer nationalen Wirtschaftszone auf 200 Seemeilen $(370 \mathrm{~km})$ auf Basis des Seerechtsübereinkommens der Vereinten Nationen an, wodurch jene Anrainerstaaten größere Freiheiten in der wirtschaftlichen und sogar militärischen Nutzungen der Arktis erhalten (Görner, 2018). Unter diesen Voraussetzungen könnte der für die Forschung frei zugängliche Raum eingeschränkt werden. Die Wissenschaft sollte daher rechtzeitig ihre Stimme nutzen und ihren Einfluss auf die Politik geltend machen, um darauf hinzuwirken, dass zur Erfüllung der Ziele in den Arktisleitlinien eine freie Polarforschung (auch bei Ausdehnung des Festlandsockels) garantiert wird.

\section{Schlussbetrachtung}

Die im August 2019 veröffentlichten neuen Leitlinien deutscher Arktispolitik sind in mehrfacher Hinsicht ein besonderes Dokument der deutschen Außenpolitik. Sie legen als zentrales Strategiepapier die Nutzungsziele Deutschlands in der Arktis dar, welche wiederum auf einem breitangelegten Themenspektrum aufbauen. Gleichzeitig betonen sie die besondere Rolle der deutschen Arktisforschung, auf deren ausgesprochen langjähriger Expertise basierend, sich Deutschland als ,internationaler Akteur im Hohen Norden“ definiert und deren Forschungsergebnisse die Grundlage für sämtliche arktispolitische Handlungen der Bundesregierung sein sollen.

Der vorliegende Beitrag hat diese Schlüsselrolle der Wissenschaft bei der künftigen Umsetzung der Arktisleitlinien näher betrachtet. Es wurde herausgearbeitet, dass eine be-

\footnotetext{
${ }^{6}$ Bei der UN-Kommission zur Begrenzung des Festlandsockels (CLCS) haben folgende Länder Anträge zur Erweiterung ihrer Wirtschaftszone gestellt: Russland (2001/2015), Norwegen (2006), Kanada (2013), Dänemark (2014). Bislang wurde nur der norwegische Antrag positiv beschieden.
} 
sonders enge Zusammenarbeit zwischen Politik und (ArktisForschung besteht, aus der ein Wissenstransfer entstanden ist, der für die erfolgreiche Zielerreichung der Arktisleitlinien unerlässlich erscheint. Beispielhaft haben sich verschiedene Formate wie etwa der Arktisdialog etabliert, mithilfe derer Forschungswissen, Erfahrungen und internationale Expertise nicht nur innerhalb des Wissenschaftssystem zur Verfügung gestellt, sondern auch in Gesellschaft und Politik kommuniziert werden. Darüber hinaus wurden mehrere bekannte Wissensträger*innen der Arktisforschung aus verschiedenen Forschungseinrichtungen betrachtet, die aufgrund ihres unterschiedlichen wissenschaftlichen Hintergrundes vielfältige Lösungsansätze bieten können, um auf die Zielerreichung der Arktisleitlinien hinzuwirken. Es wurde die Ansicht vertreten, dass sich die Arktisforschung trotz ihrer ursprünglichen naturwissenschaftlichen Verwurzelung zu einer disziplinübergreifenden Integrationswissenschaft entwickelt hat. So nutzen die verschiedenen Teilbereiche, die unterschiedlichen Wissenschaftsdisziplinen, jeweils eigene Methoden zur Generierung von neuem Wissen (Systemwissen, Orientierungswissen, Transformationswissen) zur Beantwortung von arktisrelevanten Fragen. Anstatt sich nur auf eines dieser Teilgebiete zu konzentrieren, soll mit dieser Arbeit für eine noch stärkere Verknüpfung plädiert werden, um mittels eines disziplinübergreifenden Forschungsansatzes die heterogenen, komplexen und anspruchsvollen Ziele der neuen Arktisleitlinien zu forcieren.

Datenverfügbarkeit. Für diesen Artikel wurden keine Datensätze genutzt.

Interessenkonflikt. Die Autor*innen erklären, dass kein Interessenkonflikt besteht.

Danksagung. Das vorliegende Thema wurde mit wertvollen Anregungen von Volker Rachold, Donovan Dennis, Philip Matuschka sowie Michael Wenger geschrieben. So wurden dem Autor dankenswerterweise durch die Bereitstellung von Informationen und zusätzlichen, themenbezogenen Literaturbeiträgen wichtige Impulse während des gesamten Schreibprozesses gegeben. Volker Rachold, Donovan Dennis, Maria Weigel, und Philip Matuschka sei zudem für die kritische Durchsicht des Beitrages gedankt. Ein weiteres Dankeschön auch an Renate Treffeisen für die Bereitstellung des Titelbildes „Leitlinien deutscher Arktispolitik“.

Begutachtung. This paper was edited by Donovan Dennis.
Literatur

Auswärtiges Amt: Leitlinien deutscher Arktispolitik: Verantwortung übernehmen. Vertrauen schaffen. Zukunft gestalten, Zarbock, Berlin, 2019.

Blotevogel, H. H. und Wiegand, T. S.: Zur Evaluation von Wissensgenerierung und Wissenstransfer in der Akademie für Raumforschung und Landesplanung (ARL), Leibniz-Forum für Raumwissenschaften, Raumforschung und Raumordnung, 73, Hannover, 155-165, 2015.

Bothe, M.: Umweltschutz als Aufgabe der Rechtswissenschaft. Völkerrecht und Rechtsvergleichung, Zeitschrift für ausländisches öffentliches Recht und Völkerrecht, 32, C. H. Beck, München, 483-515, 1972.

Bundesamt für Naturschutz (BfN): Interessenbekundungsverfahren UFOPLAN 2018, online aufrufbar: https://www.bfn.de/fileadmin/BfN/foerderung/Dokumente/ Ufoplan_2018/3518_83_1100_Arktis_Meeresschutz.pdf (letzter Zugriff: 10. November 2020), 2018.

Cordis: Forschungsergebnisse der EU: Interviews (2009): Die Wissenschaft als ein Werkzeug der internationalen Diplomatie, online aufrufbar: https://cordis.europa.eu/article/id/ 30532-science-as-a-tool-for-international-diplomacy/de, letzter Zugriff: 10. Mai 2020.

Deutscher Bundestag: Drucksachen: Nordatlantische Versammlung. 15. Jahrestagung vom 5. bis 21. Oktober 1969, online aufrufbar: http://dipbt.bundestag.de/doc/btd/06/001/0600191. pdf (letzter Zugriff: 10. Mai 2020), 1969.

Deutscher Bundestag: Drucksache 19/13193: Antwort der Bundesregierung auf die Kleine Anfrage der Abgeordneten Andrej Hunko, Hubtertus Zdebel, Lorenz Gösta Beutin, weiterer Abgeordneter und der Fraktion DIE LINKE., online aufrufbar: https: //dip21.bundestag.de/dip21/btd/19/153/1915326.pdf (letzter Zugriff: 10. November 2020), 2019.

Deutsches Arktisbüro: Fact Sheet. Schifffahrt in der Arktis, online aufrufbar: https://www.arctic-office.de/fileadmin/user_upload/ www.arctic-office.de/PDF_uploads/FactSheet_Schifffahrt.pdf (letzter Zugriff: 10. May 2020), 2019a.

Deutsches Arktisbüro: Fact Sheet. Arktischer Rat, online aufrufbar: https://www.arctic-office.de/fileadmin/user_upload/ www.arctic-office.de/PDF_uploads/Arktischer_Rat/Factsheet_ Arktischer_Rat.pdf (letzter Zugriff: 10. Mai 2020), 2019b.

Ecologic Institute: Arctic Summer College, online aufrufbar: https: //www.arcticsummercollege.org/, letzter Zugriff: 10. Mai 2020.

Elsner, H., Sievers, H., Szurlies, M., und Wilken, H.: Das mineralische Rohstoffpotential der Arktis, Commodity Top News, 41, Bundesanstalt für Geowissenschaften und Rohstoffe (BGR), Hannover, 1-12, 2014.

European Environment Agency (EEA): The melting Arctic, online aufrufbar: https://www.eea.europa.eu/articles/the-melting-arctic (letzter Zugriff: 10. Mai 2020), 2019.

Görner, G.: Die Arktis und das Seevölkerrecht, Welttrends, Das Außenpolitische Journal, 140, Potsdamer Wissenschaftsverlag, Potsdam, 51-56, 2018.

Grosfeld, L., Lenz, J., Fugmann, G., Gunnarsson, P., Níelsson, E., and Rachold, V.: Raising awareness and building capacity for science-based policy-making. Workshop Summary, online aufrufbar: https://www.arctic-office.de/fileadmin/user_upload/ 
www.arctic-office.de/PDF_uploads/Science_Policy_Report_ 2020.pdf (letzter Zugriff: 10. November 2020), 2019.

Hansa: International Maritime Journal: Flotte groß, Flagge klein, 156, Schifffahrts-Verlag HANSA, Hamburg, 30-31, 2019.

Heinemann, G., Braun, M., Brey, T., Damaske, D., Melles, M., Rhein, M., und Willmes, S.: Polarforschungsagenda 2030. Status und Perspektiven der deutschen Polarforschung. Statusbericht des Deutschen Nationalkomitees SCAR/IASC der Deutschen Forschungsgemeinschaft, online aufrufbar: https://epic. awi.de/id/eprint/45578/1/Polarforschungsagenda2030.pdf (letzter Zugriff: 10. November 2020), 2017.

Institut für transformative Nachhaltigkeitsforschung (IASS): Forschungsgruppe Governance der Arktis, online aufrufbar: https://www.iass-potsdam.de/de/forschungsgruppe/ governance-der-arktis, letzter Zugriff: 10. Mai 2020.

Intergovernmental Panel on Climate Change (IPCC): Special Report. The Ocean and Cryosphere in a Changing Climate, online aufrufbar: https://www.ipcc.ch/site/assets/uploads/sites/ 3/2019/12/SROCC_FullReport_FINAL.pdf (letzter Zugriff: 10. Mai 2020), 2019.

Lenhard-Schramm, N.: Was ist wissenschaftlich?, in: Das Zentrum für Wissenschaftstheorie. Dokumentation der Aktivitäten in den Jahren 2006 bis 2012, herausgegebon von: Köhler, T. A., Zentrum für Wissenschaftstheorie, Münster, 159-161, 2012.

Maribus: 6. World Ocean Review. Arktis und Antarktis. Extrem, klimarelevant, gefährdet, online aufrufbar: https://worldoceanreview.com/wp-content/downloads/wor6/ WOR6_de.pdf (letzter Zugriff: 13. November 2020), 2019.

McCallum, A.: Polar science needs a foundation. Where is the research into polar infrastructure?, Adv. Polar Sci., 28, 1-2, 2018.

Meereisportal: Titelseite der Leitlinien deutscher Arktispolitik der Bundesregierung. Kurzmeldung vom 30. August 2019, online aufrufbar: https://www. meereisportal.de/archiv/2019-kurzmeldungen-gesamttexte/ bundesregierung-beschliesst-neue-leitlinien-der-arktispolitik/ (letzter Zugriff: 20. Mai 2020), 2019.

Mittelstraß, J.: Der Flug der Eule. Von der Vernunft der Wissenschaft und der Aufgabe der Philosophie, Suhrkamp, Frankfurt am Main, 1989.
Notz, D.: Die Arktis im Klimawandel, in: Aus Politik und Zeitgeschichte (APuZ), Bundeszentrale für Politische Bildung, online aufrufbar: https://www.bpb.de/apuz/33507/ die-arktis-im-klimawandel? $\mathrm{p}=0$ (letzter Zugriff: 6. November 2020), 2011.

Osipov, I., Radford, G., Aksnes, D., Kullerud, L., and Hirshberg, D.: Digital Science Report. International Arctic Research Analyzing Global Funding Trends A Pilot Report, online aufrufbar: https://digitalscience.figshare.com/articles/International_ Arctic_Research_Analyzing_Global_Funding_Trends_A_Pilot_ Report_2017_Update_/4829455 (letzter Zugriff: 10. Mai 2020), 2017.

Pohl, C. und Hirsch Hadorn, G.: Gestaltungsprinzipien für die transdisziplinäre Forschung, Oekom, München, 2006.

Pohl, C. und Hirsch Hadorn, G.: Methodenentwicklung in der transdisziplinären Forschung, in: Transdisziplinäre Forschung. Integrative Forschungsprozesse verstehen und bewerten, herausgegeben von: Bergmann, M. und Schramm, E., Campus, Frankfurt, 69-92, 2008.

Rost, F.: Lern- und Arbeitstechniken für das Studium, Springer, Wiesbaden, 1966.

Schmidt, J. C.: Dimensionen der Interdisziplinarität. Wege zu einer Wissenschaftstheorie der Interdisziplinarität, Zeitschrift für Technikfolgenabschätzung in Theorie und Praxis, 2, 12-17, 2005.

Umweltbundesamt (UBA): Neue Leitlinien deutscher Arktispolitik, online aufrufbar: https://www.umweltbundesamt.de/themen/ neue-leitlinien-deutscher-arktispolitik (letzter Zugriff: 10. Mai 2020), 2019.

Venzke, J. F.: Vor 120 Jahren. Die erste deutsche NordpolarExpedition, Polarforschung, 58, Deutsche Gesellschaft für Polarforschung, Bremerhaven, 47-51, 1988.

Voßkuhle, A.: Das Konzept des rationalen Staates, in: Governance von und durch Wissen, herausgegeben von: Schuppert, G. F. und Vosskuhle, A., Nomos, Baden-Baden, 2008. 